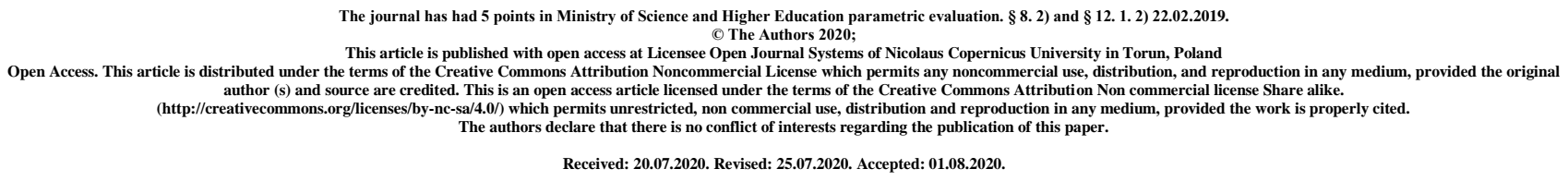

\title{
Effect of immunomodulating treatment (usage of DMARDs) in rheumatoid arthritis on depression
}

\author{
Magdalena Kozyra ${ }^{1}$, Patryk Zimnicki ${ }^{1}$, Natalia Śmiech ${ }^{1}$, Justyna Kaczerska ${ }^{1}$, Martyna \\ Nowińska', Joanna Milanowska²
}

(1) Student Research Group of Applied Psychology, Medical University of Lublin

(2) Department of Applied Psychology, Medical University of Lublin

*Address for correspodence: Staffa 24, 20-454 Lublin, telephone number: 695562174, e-mail address: madzia.kozyra.1221@ poczta.onet.pl

ORCID ID and E-mail

Magdalena Kozyra https://orcid.org/0000-0002-0260-4102 madzia.kozyra.1221 @ poczta.onet.pl

Patryk Zimnicki https://orcid.org/0000-0002-5808-8661 patryk.zimnicki.97@o2.pl

Natalia Śmiech https://orcid.org/0000-0002-8920-7969 natalia.smiech@gmail.com Justyna Kaczerska https://orcid.org/0000-0003-2642-9377 justynakaczerska@ gmail.com

Martyna Nowińska https://orcid.org/0000-0002-8167-698X m.nowinska98@ gmail.com Joanna Milanowska https://orcid.org/0000-0001-9741-1583 joannamilanowska@ umlub.pl 


\section{ABSTRACT}

Introduction: There are similarities in the level of cytokines in Rheumatoid Arthritis (RA) and depression. That is why, during usage of Disease-modifying antirheumatic drugs (DMARDs) which modulate the the level of cytokines may influence both depression and RA

The aim of the study: The aim of this article is to find out in what way do the DMARDs influence the depression.

Material and method: The research of the scientific articles was made on a database of PubMed and Google Scholar.

Description of the state of knowledge: The results show, that most of the DMARDs may decrease the level of depression what is connected with the way of how these medicines influence the level of cytokinese. There are some exceptions such as Chloroquine and anakinra which will increase the probability of depression and there is adalimumab where the is case report which presents one case of appearance of depression after taking this medicine.

Summary: This article gathers scientific research on the influence of DMARDs on depression. It shows the mechanisms, and possible ways in which these medicins influence both RA and depression. For some of the DMARDs there are no scientific articles which would include their influence on depression and it might be the way of further researches.

Key words: Rheumatoid Arthritis, Disease-modifying antirheumatic drugs, depression;

\section{Introduction}

Although the causes of depression are unfortunately not sufficiently understood, it is said that its occurrence may be due to, among others, genetic predisposition, difficult childhood events, current stress, biologically conditioned sensitivity to the seasonal deficit of sunlight or certain personality traits such as pessimism, self-disbelief, excessive criticism towards oneself and others, perfectionism, excessive dependence on the approval and acceptance of other people, inability to express anger and direct it outside [1]. The association of biological mechanisms of depression with chronic stress undermines the traditional division of the disease [2]. The new concepts contain intuitions that depression is a disease of the body with significant disturbances in the mechanisms of human biology, but also a disease associated with difficult life events or problems in achieving life goals. Since the etiology of depression is complex, the terms that indicate the type of depression allegedly caused by one cause should disappear from the medical language. Concepts such as endogenous depression, psychogenic depression, and somatogenic depression are particularly confusing. Unfortunately, these inaccurate terms are deeply rooted in the Polish psychiatric and psychological tradition, and are still used, which leads to misunderstandings.

In turn, rheumatoid arthritis is a chronic inflammatory disease that affects the joints. The essence of the disease is the inflammatory process that begins inside the joint. An unknown factor stimulates the synovial membrane that lines the joint to an inflammatory response. It enlarges and destroys the adjacent structures - cartilage, bones, ligaments and tendons [3]. This is manifested initially by pain and swelling, followed by irreversible damage and loss of mobility of the joint. Inflammation of the cartilage and other structures of the joint favors the formation of secondary degenerative changes. The development of rheumatoid arthritis is increased by various injuries to the 
locomotion system, chronic infections, working in cold, wet conditions, etc. A characteristic symptom of RA is also the presence of subcutaneous, painless nodules, occurring in places of increased pressure, such as the ulnar process of the humerus. The causes of RA include, among others, hereditary burden, female gender, infection with certain pathogens, smoking, stress or a defect in the immune system [4], which leads to the misidentification of one's own tissues as an "enemy" and the production of autoantibodies to destroy them. Several genes responsible for this process have been identified (including the HLA DRB1 histocompatibility genes) [4]. RA affects the results of laboratory tests, namely the ESR, leukocytosis, serum iron concentration, protein composition and Waaler-Rose test change.

\section{Materials, methods and aim}

Scientific publications were found regarding the relationship between the DMARDs used in the treatment of RA and the depression. PubMed and Google Scholar detabases were used to find these Scientific articles.

\section{Characteristics of Disease-modifying antirheumatic drugs}

The introduction of biological drugs in the treatment of rheumatic diseases has opened a new era in the treatment of such diseases as rheumatoid arthritis (RA), psoriatic arthritis or ankylosing spondylitis. These drugs are highly effective, occasionally cause side effects, and can even be taken by pregnant women. There are also biosimilar drugs, whose effectiveness does not differ significantly from classic biological drugs. About $70 \%$ of patients show a positive response to treatment.

Disease-modifying antirheumatic drugs (DMARDs) are essential in the treatment of RA. They prevent or delay destructive changes in the joints. The most important of these is methotrexate, which is the first-line drug. The immunosuppressive and anti-inflammatory effects of methotrexate are associated with the inhibition of folate-dependent enzymes by polyglutamate derivatives, or indirectly with the release of adenosine and/or apoptosis and clonal deletion of activated peripheral blood lymphocytes in the $\mathrm{S}$ phase [5].

Leflunomide, sulfasalazine, and anti-malarial drugs (hydroxychloroquine, chloroquine) are the next-line (after methotrexate) conventional synthetic drugs for the treatment of RA.

Leflunomide has immunomodulatory and immunosuppressive properties, it acts in antiproliferative and anti-inflammatory way. The leflunomide metabolite A771726 is probably responsible for the therapeutic effect. It inhibits dihydroorotate dehydrogenase activity, which leads to inhibition of de novo synthesis of uridine monophosphate ribonucleotide. It exhibits antiproliferative properties, inhibits COX-2 activity, and in concentrations significantly higher than therapeutic, inhibits tyrosine kinase during cell division [6].

The mechanism of action of sulfasalazine is not clearly explained. It exhibits immunosuppressive properties mainly within connective tissue, intestinal wall and in serous fluids, where it reaches the highest concentrations. The effects of sulfasalazine metabolites acting locally in the intestinal wall and systemically are better known. Sulfapridine presumably inhibits cytotoxic leukocyte function and lymphocyte transformation. The anti-inflammatory effect of 5-aminosalicylic acid (mesalazine) probably plays the most important role in the treatment of inflammatory bowel 
disease. This effect is primarily local and consists in the inhibition of cyclic oxygenase and lipid oxygenase, which prevents the synthesis of prostaglandins, leukotrienes and other mediators of inflammation. It probably also binds free oxygen radicals [7].

In the treatment of early RA, anti-malarial drugs are used - chloroquine and hydroxychloroquine, whose mechanism of action is also not fully understood. These drugs interfere with lysosomal activity and autophagy, affect membrane stability and change signaling pathways and transcriptional activity, which may result in inhibition of cytokine production and modulation of some co-stimulatory molecules [8].

However, many commonly used DMARDs: hydroxychloroquine, gold salts, sulfasalazine, and methotrexate) are highly toxic, and after a long time, most patients will have a reduced response to treatment. These drugs slow down but do not inhibit the progression of destructive changes in the joints.

The introduction of infliximab, etanercept and adalimumab interfering in immune phenomena occurring in RA in the years 1998-2003 was a significant advance and a breakthrough in the treatment of the disease. $[9,10]$. The action of these biopharmaceuticals is directed against tumor necrosis factor $\alpha$ $(\mathrm{TNF}-\alpha)$. This cytokine is responsible for the production of other proinflammatory cytokines, stimulates the production of adhesion molecules, enhances angiogenesis and the release of inflammatory mediators. TNF- $\alpha$ is present in high concentrations within inflammatory joints in the course of RA. Currently, other biological drugs with anti-cytokine and non-anti-cytokine activity are also being used. The first group includes: adalimumab, anakinra, certolizumab, etanercept, golimumab, infliximab, tocilizumab.

Anakinra is a recombinant interleukin-1 (IL-1) receptor antagonist with anti-inflammatory and immunomodulatory activity, used to treat rheumatoid arthritis and other inflammatory inflammation. Anakinra is associated with a low rate of increase in serum enzyme activity during treatment and in rare cases of clinically apparent acute liver damage [11].

Golimumab is a human monoclonal antibody that forms stable complexes with high affinity for the soluble and transmembrane form of human TNF- $\alpha$, preventing binding of TNF- $\alpha$ to its receptors. Binding of human TNF by golimumab neutralizes TNF- $\alpha$ induced cell surface expression of the Eselectin adhesion molecule, intercellular adhesion molecule (VCAM-1) and intercellular adhesion molecule (ICAM-1) of endothelial cells [12].

Certolizumab is a new TNF inhibitor, consisting of a humanized Fab fragment combined with a $40 \mathrm{kD}$ polyethylene glycol fragment (PEG). This unique structure can avoid potential Fc-mediated effects seen in vitro, such as complement-dependent or antibody-dependent cellular cytotoxicity or apoptosis [13].

Toclizumab is a recombinant, humanized $\mathrm{IgG}_{1}$ monoclonal antibody. It binds specifically to IL-6 receptors (sIL-6R and mIL-6R), both soluble and membrane-bound, thereby inhibiting signal transduction by the mediated pathway through these receptors; IL-6, which is a pleiotropic proinflammatory cytokine, is weakened [14]. 
Representatives of non-anti-cytokine biologics include rituximab and abatacept.

Rituximab is an anti-B cell antibody involved in inflammatory processes in the course of autoimmune diseases [15].

Although the development of disease-modifying anti-rheumatic drugs (DMARDs) and tumor necrosis factor antagonists has improved the clinical outcomes of many patients with rheumatoid arthritis (RA), a significant proportion of patients have an inadequate response. Because $\mathrm{T}$ cells promote numerous pathways in RA, these cells are the logical target of anti-inflammatory therapy. Abatacept (CTLA-4Ig) is a soluble recombinant fusion protein that competes with CD28 for binding to CD80 and CD86 and can therefore be used to selectively modulate T cell activation [16].

The choice of drug depends on the activity and duration of the disease, previous treatment, prognostic factors (worse prognosis: autoantibodies [RF and / or ACPA, especially in high-profile], very high disease activity, early appearance of erosions), concomitant diseases and contraindications, adverse reactions and drug availability (preparations, dosage, contraindications and principles for monitoring treatment).

\section{Immunological dependence of proinflammatory cytokines in RA on depression}

Every third person with RA suffers from mood problems. In most cases, they are depression and anxiety disorders [17]. From an immunological point of view, elevated TNF-alpha, INF-alpha, IL-6, IL-10, and lower IL-1 levels are administered during depression. The given relationships in cytokine levels are observed in RA. Hence the large relationship between RA and depression. It may be associated with these similar immune conditions of both disease entities. Moreover, if the RA patient had no symptoms of depression, one would be more likely to experience remission. Similarly, patients who were in remission showed less symptoms of depression. From this one can deduce a high affinity for both diseases [19].

The mechanism of the dependence of cytokines on depression is associated, for example, with the fact that pro-inflammatory cytokines whose level is increased in depression (TNF-alpha, IL-2, IL-6) reduce the level of dopamine in the blood, which in turn leads to the pathomechanism of depression. Another example is the increase in serotonin reuptake in the presence of increased levels of proinflammatory cytokines such as IL- $1 \beta$, TNF- $\alpha$, and IFN- $\alpha$ [19].

\section{Biological drugs}

Anakinra

One of the original biological drugs used in rheumatoid arthritis is anakinra - an interleukin 1 (IL-1Ra) inhibitor. In stressful situations, mRNA is produced in the brain which is responsible for IL-1 synthesis. It was proved that the group of mice lacking IL-1Ra there was no inhibition of IL-1 and this group of mice showed greater antidepressant behavior. Moreover, in the group of mice with an excess of IL-1Ra, more anxiety behaviors were observed in elevated plus-maze (EPM) tests [20].

Observations, also indicative of the effect of IL-1Ra on prodepressive effects, were noted by Ovaskainen Y at al. Researchers have reported that middle-aged men who suffer from depression have significantly increased IL-1Ra levels and the IL-1Ra to IL-1 ratio compared to a healthy control [21]. Milaneschi $\mathrm{Y}$ at al. Came to similar conclusions. examining IL-1 and IL-1Ra levels for six years, in a 
study of almost 1,000 participants, stating that people suffering from depression have elevated IL-1Ra levels [22].

Another experiment has attempted to show an antidepressant effect on rats using IL-1Ra for depression occurring at seizures. However, no effect was achieved in this experiment and the IL-1Ra part of the study was removed from the study [23].

Considering the above studies, it can be concluded that the use of the biological drug anakinra, which is IL-1Ra, can cause a patient with depressive disorders.

\section{Adalimumab}

Another original biological drug used in RA treatment is adalimumab, which is a recombinant human monoclonal antibody directed against TNF- $\alpha$. Studies say that elevated TNF- $\alpha$ levels co-occur with depression, but not only. Elevated TNF- $\alpha$ levels are also mentioned in schizophrenia and bipolar disorder [24]. In turn, Adalimumab inactivating TNF- $\alpha$ reduces its effect [25].

Confirmation of this is certainly several studies assessing the mental state and depressionrelated disorders in patients taking this drug. For example, one study in its third stage of a randomized, double-blind study observed in people taking adalimumab at a dose of 40mg weekly treated for Crohn's disease, significantly less symptoms of depression than the control. This effect was maintained throughout the duration of the study - that is, for 56 weeks [26]. Patients in a randomized, double-blind study looked at the effect of adalimumab on depressive symptoms in patients with psoriasis at the same dose. Also in this study, the positive effect of the drug on depression symptoms was confirmed, which was measured on the Zung Self-rating Depression Scale (ZDS) [27].

Despite the anti-depressant effect of adalimumab, it is worth noting that the case with the reverse effect of this drug has been described. A 56-year-old patient treated for rheumatoid arthritis, after unsuccessful treatment with matotrexate, received a switch to adalimumad. Initially, she received $40 \mathrm{mg}$ of the drug under the skin for 14 days. After remission of the disease, it was decided to increase the frequency of injections every 7 days for $40 \mathrm{mg}$ of adalimumab. Two weeks after increasing the dose, the woman began to complain of a rash, blisters in the mouth, and suicidal thoughts. She had never reported similar mental symptoms before. After immediate change to glucocorticosteroids, the patient's suicidal thoughts resolved [28]. This case shows that although most studies indicate a positive antidepressant effect of adalimumab, it should be remembered that patients may have the opposite effect.

\section{Etanercept}

Etanercept - a recombinant p75 receptor protein that binds to TNF- $\alpha$ blocking its effects in mice showed a significantly increased risk of anxiety behavior than the control group. In turn, it was found that the same group of mice receiving this drug had reduced cognitive function compared to the control group. A reduced number of microglia within the hippocampus [29] was also observed. In turn, a crosssectional observational study conducted by Taiwanese scientists found a lower risk of developing depression in patients taking etanercept, but no effect on anxiety disorders, compared to the control group not taking any biological drugs [30]. 


\section{Imfliximab}

Like etanercept, imfliximab blocks the action of the TNF- $\alpha$ cytokine. Therefore, one could immediately predict a similar effect of this drug on depression in patients suffering from RA. Comparative study of monoteraphy of methotrexate vs. infliximab showed that both groups of patients taking one or the other drug had a lower likelihood of depression. Given the Hamilton Rating Scale for Depression (HAM-D) and Disease Activity Score (DAS), there was no statistical difference between them [31,32], and on the Self-Rating Depression Scale (SDS) there was a statistical difference between them in favor of infliximab [32]. However, in RA therapy alone, imfliximab was significantly more effective than monotrexate alone [31].

\section{Tocilizumab}

Tocilizumab is the first human monoclonal antibody directed against IL-6 receptor. In the literature, IL-6 is of great importance in the development of the pathomechanism of RA, but not only. There is a known relationship between increased levels of pro-inflammatory IL-6 and the risk of depression [33]. It is no wonder then that research indicates the combined effects of tocilizumab not only on RA but also on depression [34]. However, there is also a voice from experts who says that taking tocilizumab may not be an appropriate therapeutic goal in the treatment of depression [35], so opinions are somewhat divided.

\section{Synthetic drugs}

\section{Methotrexate}

Methotrexate, used not only in RA, but also in a number of other diseases, and above all as a cytostatics in numerous cancers, from an immunological point of view it inhibits the activation of $\mathrm{T}$ lymphocytes, lowers the level of B lymphocytes and inhibits the binding of IL-1-beta to its superficial receptors. In depression, there is an increase in $\mathrm{T}$ lymphocytes in patients with major depressive disorder (MDD) compared to the control group [36].

The use of methotrexate or leflonomide is associated with less suicidal thinking among patients with RA than in those using hydroxychloroquine or biological drugs [37]. The same study found that depression was most prevalent in RA patients using biological medications, which implies that methotrexate is more effective than them in combating depression. Similar results were obtained by Sergeant JC et al. of the 1,050 people enrolled in the study, 449 did not respond to methotrexate treatment. These people showed a higher tendency to anxiety disorders and depression [38]. The literature also includes a case report of two children diagnosed with mood disorders, linking them directly with treatment with methotrexate to two different dermatological diseases [39].

\section{Leflunomide}

Leflunomide, in addition to the inhibitory effect of the mitochondrial enzyme dihydroorotate dehydrogenase (DHODH) and the synthesis of uridine monophosphate (rUMP), thanks to which it regulates, or actually inhibits the growth and production of lymphocytes, it is also an MAO-A and MAO-B inhibitor. And although regulation of both MAO isoforms is not a major leflunomide pathway, inhibition is used to treat depression. Hence, the conclusion that leflunomide, by means of MAO inhibition, may, just like classic drugs used, be involved in the treatment of depression [40]. 
Chinese scientists have decided to compare leflunomide to the newly discovered Chinese herbal drug Xinfeng, which has proved to be used in the treatment of RA. The mechanism of action of Xinfeng is probably based on the modulation of TGF- $\beta 1$ / Smads, Notch-Jag-ged / Delta. Thanks to this drug affects the level of Treg, IL-1b, TNF- $\alpha$, IL-4 and IL-10 lymphocytes. In a randomized, doubleblind study, patients were divided into two groups. The treatment group received Xinfeng and placebo for leflunomide, and the control group received placebo for Xinfeng and leflunomide. The results indicated a significant reduction in depression calculated on the self-rating depression scale (SDS) in the treatment group compared to the control group [41].

\section{Sulfasalazine}

Sulfasalazine, by inhibiting local PGE2 prostaglandins, leukotrienes and increased IgA and IgG production, has a largely immunomodulatory nature. In addition, sources have reported that it may lower B cell levels in their early stages of formation, differentiation and proliferation [42].

In mice, sulfasalazine treatment showed a very similar antidepressant effect as the selective serotonin reuptake inhibitors (SSRIs) used in the control group. However, this effect has been tested in the treatment of cancer cells and the theory of researchers is that prevention of depressive behavior using sulfasalazine is more possible due to its inhibition of releasing of glutamine of the tumor [43]. It is therefore unknown whether this or that mechanism would affect depression during RA treatment.

\section{Chloroquine}

The antiprotozoal drug commonly used in the treatment of malaria is also used in the treatment of RA. Unlike other biological or even synthetic drugs used in this disease, chloroquine has confirmed cases of prodepressive effects. And although there are many theories on this subject, it may be related to its inhibition of IL-1 expression, which is reduced in levels of depressed people [21]. In addition to the effect of chloroquine on IL-1, its effect on inhibition of IL-6 and TNF-alpha expression is also given. The increase in these two cytokines is again associated with the risk of depression and RA. From this it follows that due to this effect, chloroquine can be used to treat RA and depression. However, this seems ineffective when it comes to that second disease [44]. In addition to depression, chloroquine can cause a number of other psychological symptoms such as insomnia, aggression, mania, hyperactivity, hallucinations and even suicidal thoughts. They are probably associated with changes in neuronal neurotransmission causing such effects [45].

\section{Remission of RA by taking a SSRI (escitalopram)}

There is one case report in which there is information about a 60-year-old man struggling with RA for years. In the past, he took a number of different biological and synthetic drugs used to treat RA. However, there was no remission. Several years ago, he had several episodes of depression. He has never performed before. At the time of very severe depression, he consulted a psychiatrist, from whom he received escitalopram and risperidone. It turned out that during depression therapy, his RA symptoms significantly decreased and they could even be qualified for remission. Moreover, after discontinuing psychiatric drugs, there was a rapid deterioration in both mental and exacerbation of RA. The re-admission of antidepressant drugs meant that both depression and RA are under constant control [46]. 


\section{Summary}

At the immunology level, RA and depression have a lot in common. Numerous literature and studies report the co-occurrence of both these diseases. This is also confirmed by the fact that drugs that act on RA also treat depression symptoms. There are no studies on all immunomodulatory drugs biological and synthetic, however, those studies that check the symptoms of depression when using these drugs unanimously say that these drugs can reduce depressive behavior.

However, one should remember about the unique possibilities of just the opposite. An example of this is the case report of a 56-year-old woman in whom adalimumab was the direct cause of suicidal thoughts. They appeared immediately after increasing the dose of the drug, and resolved as soon as the rheumatoid arthritis treatment was changed to glucocorticosteroids [28]. The literature also gives many examples that chloroquine used in the treatment of malaria can induce depression [44,47]. Although it is not such a common drug used in RA, it is still possible to use. And although it can improve the condition of a patient with RA, it can aggravate or even trigger depression, to which such a patient is particularly vulnerable. People who suffer from RA will receive anakinra, which increases the level of IL-1Ra, thus lowering the level of IL-1 [20,21,22,23], are much more exposed to depression. In a similar way, chloroquine can induce depression, because it inhibits IL-1 production by lowering its level.

\section{References}

[1] Bernaras E, Jaureguizar J, Garaigordobil M. Child and Adolescent Depression: A Review of Theories, Evaluation Instruments, Prevention Programs, and Treatments. Front Psychol. 2019 Mar 20;10:543. doi: 10.3389/fpsyg.2019.00543. PMID: 30949092; PMCID: PMC6435492.

[2] Slavich GM, Irwin MR. From stress to inflammation and major depressive disorder: a social signal transduction theory of depression. Psychol Bull. 2014 May;140(3):774-815. doi: 10.1037/a0035302. Epub 2014 Jan 13. PMID: 24417575; PMCID: PMC4006295.

[3] Möller B, Kollert F, Sculean A, Villiger PM. Infectious Triggers in Periodontitis and the Gut in Rheumatoid Arthritis (RA): A Complex Story About Association and Causality. Front Immunol. 2020 Jun 3;11:1108. doi: 10.3389/fimmu.2020.01108. PMID: 32582191; PMCID: PMC7283532.

[4] Deane KD, Demoruelle MK, Kelmenson LB, Kuhn KA, Norris JM, Holers VM. Genetic and environmental risk factors for rheumatoid arthritis. Best Pract Res Clin Rheumatol. 2017 Feb;31(1):318. doi: 10.1016/j.berh.2017.08.003. Epub 2017 Sep 18. PMID: 29221595; PMCID: PMC5726551.

[5] Renata Budzyńska, Helena Zakliczyńska, Methotrexate in the treatment of the patients with rheumatoid arthritis, Postepy Hig Med Dosw (online), 2004; 58: 108-117

[6] Baza Leków. Leflunomid - Medycyna Praktyczna: opis, ceny, refundacja. https://www.mp.pl/pacjent/leki/subst.html?id=4321.

[7] "Baza Leków." Medycyna Praktyczna: opis, ceny, refundacja.

https://www.mp.pl/pacjent/leki/leki/subst.html?id=737.

[8] Schrezenmeier E, Dörner $\mathrm{T}$ Mechanisms of action of hydroxychloroquine and chloroquine: implications for rheumatology, Nat Rev Rheumatol. 2020 Feb 7, PMID: 32034323

[9] Culy C.R., Keating G.M.: Etanercept. An updated review of its use in rhaumatoid arthritis, psoriatic arthritis and juvenile rheumatoid arthritis. Drugs, 2002, 62(17): 2493. 
[10] Markham., Lamb H.M.: Infliximab: a review of its use in the management of rheumatoid arthritis. Drugs, 2000, 59(6): 1341.

[11] Anakinira, LiverTox: Clinical and Research Information on Drug-Induced Liver Injury [Internet]. Bethesda (MD): National Institute of Diabetes and Digestive and Kidney Diseases; 2012-2017 Jul 5. PMID: 31643927

[12] E C Keystone, M C Genovese, L Klareskog, E C Hsia, S T Hall, P C Miranda, J Pazdur, S-C Bae, W Palmer, J Zrubek, M Wiekowski, S Visvanathan, Z Wu, M U Rahman; Golimumab, a human antibody to tumour necrosis factor $\alpha$ given by monthly subcutaneous injections, in active rheumatoid arthritis despite methotrexate therapy: the GO-FORWARD Study

[13] Edward Keystone, Désireé Van Der Heijde, David Mason Jr., Robert Landewé, Ronald Van Vollenhoven, Bernard Combe, Paul Emery, Vibeke Strand, Philip Mease, Chintu Desai, Karel Pavelka, Certolizumab pegol plus methotrexate is significantly more effective than placebo plus methotrexate in active rheumatoid arthritis: Findings of a fifty-two-week, phase III, multicenter, randomized, doubleblind, placebo-controlled, parallel-group study, 30 October 2008

[14] Baza Leków. https://www.mp.pl/pacjent/leki/leki/subst.html?id=4768.

[15] Małgorzata Tłustochowicz, Rituximab: the role in the treatment of rheumatoid arthritis Reumatologia 2011; 49, 1: 55-61

[16] Piotr Wiland, Bożena Kowalewska, Eliza Roszkowska, Jacek Szechiński Review paper The role of abatacept in the treatment of rheumatoid arthritis, Reumatologia 2007; 45, 4: 205-214

[17] Murphy LB, Sacks JJ, Brady TJ, Hootman JM, Chapman DP. Anxiety and depression among US adults with arthritis: prevalence and correlates. Arthritis Care Res (Hoboken). 2012 Jul;64(7):968-76. doi: 10.1002/acr.21685.

[18] Kekow J, Moots R, Khandker R, Melin J, Freundlich B, Singh A. Improvements in patientreported outcomes, symptoms of depression and anxiety, and their association with clinical remission among patients with moderate-to-severe active early rheumatoid arthritis. Rheumatology (Oxford). 2011 Feb;50(2):401-9. doi: 10.1093/rheumatology/keq327.

[19] Lotrich FE. Inflammatory cytokine-associated depression. Brain Res. 2015 Aug 18;1617:113-25. doi: 10.1016/j.brainres.2014.06.032.

[20] Wakabayashi C, Numakawa T, Odaka H, Ooshima Y, Kiyama Y, Manabe T, Kunugi H, Iwakura Y. IL-1 receptor-antagonist (IL-1Ra) knockout mice show anxiety-like behavior by aging. Neurosci Lett. 2015 Jul 10;599:20-5. doi: 10.1016/j.neulet.2015.05.019

[21] Ovaskainen Y, Koponen H, Jokelainen J, Keinänen-Kiukaanniemi S, Kumpusalo E, Vanhala M. Depressive symptomatology is associated with decreased interleukin-1 beta and increased interleukin-1 receptor antagonist levels in males. Psychiatry Res. 2009 May 15;167(1-2):73-9. doi: 10.1016/j.psychres.2007.12.004

[22] Milaneschi Y, Corsi AM, Penninx BW, Bandinelli S, Guralnik JM, Ferrucci L. Interleukin-1 receptor antagonist and incident depressive symptoms over 6 years in older persons: the InCHIANTI study. Biol Psychiatry. 2009 Jun 1;65(11):973-8. doi: 10.1016/j.biopsych.2008.11.011 [23] Mazarati AM, Pineda E, Shin D, Tio D, Taylor AN, Sankar R. Comorbidity between epilepsy and depression: role of hippocampal interleukin-1beta. Neurobiol Dis. 2010 Feb;37(2):461-7. doi: 10.1016/j.nbd.2009.11.001 
[24] Goldsmith DR, Rapaport MH, Miller BJ. A meta-analysis of blood cytokine network alterations in psychiatric patients: comparisons between schizophrenia, bipolar disorder and depression. Mol Psychiatry. 2016 Dec;21(12):1696-1709. doi: 10.1038/mp.2016.3

[25] Huang KW, Wu MK, Hung YY. Elevated TNIP3 mRNA Expression in TNF- $\alpha$-Secreting Cells from Patients with Major Depressive Disorder. Neuroimmunomodulation. 2019;26(3):153-158. doi: $10.1159 / 000501083$

[26] Loftus EV, Feagan BG, Colombel JF, Rubin DT, Wu EQ, Yu AP, Pollack PF, Chao J, Mulani P. Effects of adalimumab maintenance therapy on health-related quality of life of patients with Crohn's disease: patient-reported outcomes of the CHARM trial. Am J Gastroenterol. 2008 Dec;103(12):313241. doi: 10.1111/j.1572-0241.2008.02175.x

[27] Menter A, Augustin M, Signorovitch J, Yu AP, Wu EQ, Gupta SR, Bao Y, Mulani P. The effect of adalimumab on reducing depression symptoms in patients with moderate to severe psoriasis: a randomized clinical trial. J Am Acad Dermatol. 2010 May;62(5):812-8.Doi:10.1016/j.jaad.2009.07.022 [28] Jafri F, Sammut A. A rare case of suicidal ideation related to Adalimumab use. Open Access Rheumatol. 2018 Aug 17;10:113-115. doi: 10.2147/OARRR.S168559

[29] Camara ML, Corrigan F, Jaehne EJ, Jawahar MC, Anscomb H, Baune BT. Effects of centrally administered etanercept on behavior, microglia, and astrocytes in mice following a peripheral immune challenge. Neuropsychopharmacology. 2015 Jan;40(2):502-12. doi: 10.1038/npp.2014.199.

[30] Ng KJ, Huang KY, Tung CH, Hsu BB, Wu CH, Lu MC, Lai NS. Risk factors, including different biologics, associated with depression and anxiety in patients with rheumatoid arthritis: a cross-sectional observational study. Clin Rheumatol. 2019 Dec 10. doi: 10.1007/s10067-019-04820-x

[31] Miwa Y, Isojima S Saito M, Ikari Y, Kobuna M, Hayashi T, Takahashi R, Kasama T, Hosaka M, Sanada K. Comparative Study of Infliximab Therapy and Methotrexate Monotherapy to Improve the Clinical Effect in Rheumatoid Arthritis Patients. Intern Med. 2016;55(18):2581-5. doi: 10.2169/internalmedicine.55.6872.

[32] Miwa Y1, Nishimi A1, Nishimi S1, Saito M1, Tokunaga T1, Yanai R1, Takahashi R1, Wakabayashi K1, Kasama T1, Hosaka M2. Combined infliximab and methotrexate treatment improves the depressive state in rheumatoid arthritis patients more effectively than methotrexate alone. Eur J Rheumatol. 2014 Dec;1(4):147-149.

[33] Choy EHS, Calabrese LH. Neuroendocrine and neurophysiological effects of interleukin 6 in rheumatoid arthritis. Rheumatology (Oxford). 2018 Nov 1;57(11):1885-1895. doi: 10.1093/rheumatology/kex391.

[34] Corominas H, Alegre C, Narváez J, Fernández-Cid CM, Torrente-Segarra V, Gómez MR, Pan FM, Morlà RM, Martínez FJR, Gómez-Centeno A, Ares LL, Molina RG, González-Albo SP, Dalmau-Carolà J, Pérez-García C, Álvarez CB, Ercole L, Terrancle MÁ; ACT-AXIS Study Group. Correlation of fatigue with other disease related and psychosocial factors in patients with rheumatoid arthritis treated with tocilizumab: ACT-AXIS study. Medicine (Baltimore). 2019 Jun;98(26):e15947. doi: 10.1097/MD.0000000000015947.

[35] Maes $\mathrm{M}^{1}$, Anderson G, Kubera M, Berk M. Targeting classical IL-6 signalling or IL-6 transsignalling in depression? Expert Opin Ther Targets. 2014 May;18(5):495-512. doi: $10.1517 / 14728222.2014 .888417$. 
[36] Mohd Ashari NS, Mohamed Sanusi SNF, Mohd Yasin MA, Che Hussin CM, Wong KK, Shafei MN. Major depressive disorder patients on antidepressant treatments display higher number of regulatory T cells. Malays J Pathol. 2019 Aug;41(2):169-176. PMID: 31427552.

[37] Pinho de Oliveira Ribeiro N, Rafael de Mello Schier A, Ornelas AC, Pinho de Oliveira CM, Nardi AE, Silva AC. Anxiety, depression and suicidal ideation in patients with rheumatoid arthritis in use of methotrexate, hydroxychloroquine, leflunomide and biological drugs. Compr Psychiatry. 2013 Nov;54(8):1185-9. doi: 10.1016/j.comppsych.2013.05.010

[38] Sergeant JC, Hyrich KL, Anderson J, Kopec-Harding K, Hope HF, Symmons DPM; RAMS CoInvestigators, Barton A, Verstappen SMM. Prediction of primary non-response to methotrexate therapy using demographic, clinical and psychosocial variables: results from the UK Rheumatoid Arthritis Medication Study (RAMS). Arthritis Res Ther. 2018 Jul 13;20(1):147. doi: 10.1186/s13075-018-16455.

[39] Bhat T, Coughlin CC. Mood changes with methotrexate therapy for dermatologic disease. Pediatr Dermatol. 2018 Mar;35(2):253-254. doi: 10.1111/pde.13406.

[40] Petzer JP, Petzer A. Leflunomide, a Reversible Monoamine Oxidase Inhibitor. Cent Nerv Syst Agents Med Chem. 2016;16(2):112-9.

[41] Liu J, Wang Y, Huang C, Xu J, Li Z, Xu L, He L, Sun Y, Wang Y, Xu S, Zhao P, Mao T, Tan B, Zhu F, Zhang P, Fang L. Efficacy and safety of Xinfeng capsule in patients with rheumatoid arthritis: a multi-center parallel-group double-blind randomized controlled trial. J Tradit Chin Med. 2015 Oct;35(5):487-98.

[42] Imai F, Suzuki T, Ishibashi T, Dohi Y. Effect of sulfasalazine on B cells. Clin Exp Rheumatol. 1991 May-Jun;9(3):259-64.

[43] Nashed MG, Ungard RG, Young K, Zacal NJ, Seidlitz EP, Fazzari J, Frey BN, Singh G. Behavioural Effects of Using Sulfasalazine to Inhibit Glutamate Released by Cancer Cells: A Novel target for Cancer-Induced Depression. Sci Rep. 2017 Jan 25;7:41382. doi: 10.1038/srep41382.

[44] Jang CH, Choi JH, Byun MS, Jue DM. Chloroquine inhibits production of TNF-alpha, IL-1beta and IL-6 from lipopolysaccharide-stimulated human monocytes/macrophages by different modes. Rheumatology (Oxford). 2006 Jun;45(6):703-10.

[45] Mohan D, Mohandas E, Rajat R. Chloroquine psychosis: a chemical psychosis? J Natl Med Assoc. 1981 Nov;73(11):1073-6.

[46] Krishnadas R, Krishnadas R, Cavanagh J. Sustained remission of rheumatoid arthritis with a specific serotonin reuptake inhibitor antidepressant: a case report and review of the literature. J Med Case Rep. 2011 Mar 19;5:112. doi: 10.1186/1752-1947-5-112.

[47] Das EM, Mohan D. Chloroquine-related depression. Indian J Psychiatry. 1981 Apr;23(2):184-5. 